\title{
Transporte de sedimentos no rio Amazonas
}

\author{
Robert H. Meade ('), Carl F. Nordin Jr. ('), William F. Curtis ('), Flavio M. Costa Rodrigues ( $\left.{ }^{2}\right)$, Car!os \\ M. do Vale $\left(^{3}\right)$ e John M. Edmond ${ }^{(4)}$
}

\section{RESUMO}

O transporte médio anual de sedimentos em sus. pensão no rio Amazonas, em Óbidos, Brasil, foi estimado, a partir de novas medições, em 0,8 a $0,9 \times 10^{9}$ ton.ano. A maior parte destes sedimentos é lançada na plataforma continental.

\section{INTRODUÇÃO}

Em estimativas publicadas nos últimos 20 anos, a quantidade de sedimentos que o rio Amazonas lança no mar varia entre 0,4 e $1,0 \times 10^{9}$ ton.ano. Estimativas publicadas por volta de 1960, quando havia ainda poucos dados conhecidos quanto à concentração de sedimentos e caudal do rio, foram da ordem de 0,9 a $1,0 \times 10^{9}$ ton ano (NEDECO, 1959; Strakhov, 1961). Estimativas publicadas em 1967 e 1968, após a obtenção de novos dados sig. nificativos, foram da ordem de 0,4 a $0,5 \times 10^{9}$ ton.ano, mais baixas que as primeiras apresentadas (Gibbs, 1967; Holeman, 1968). Dados mais elaborados, que foram recolhidos na sua maioria desde 1970 (e especialmente em 1977), sugerem que as primeiras estimativas, altas, deveriam estar mais perto da realidade.

TRANSPORTE DE SEDIMENTOS EM 1977

O transporte de sedimentos na bacia do rio Amazonas foi medido durante as estações de cheias de 1976 e 1977. O conjunto mais completo de medições foi efetuado em maio e junho de 1977, quando se acompanhou o desenvolvimento da cheia, rio abaixo, medindo- se a quantidade de sedimentos em suspensão em seis locais do canal principal e em dois dos seus principais afluentes (Fig. 1). Umá vez que o navio navegou, rio abaixo, à mesma velocidade que a onda de cheia (incluindo as estações de medição efetuadas no percurso), conseguiu-se, praticamente, acompanhar a mesma fase da cheia ao longo de cerca de $3.000 \mathrm{~km}$, desde o Peru até perto da foz do Amazonas. Isto também permitiu fazer uma idéia de como o transporte de sedimentos va. ria, rio abaixo.

As quantidades de sedimentos transportados em suspensão, em 1977, foram medidas em amostras de água do rio recolhidas pelo Navio de Pesquisas Alpha Helix, através de amostrador (integrador em profundidade) de grande capacidade. $\mathrm{O}$ amostrador integrador em profundidade é baixado até ao fundo do rio e depois içado, à velocidade constante, até à superfície; o seu bocal está dimensionado de modo a recolher a mistura de água e sedimento a velocidade idêntica à do rio. A amostra resultante é corrigida para as diferenças de velocidade ao longo da coluna de água. O transporte de sedimentos em suspensão é obtido por simples multiplicação do caudal pela concentração de sedimentos medida da amostra (Guy \& Norman, 1970; Porterfield, 1972). Em cada um dos locais indicados na Tabela 1, recolheram-se amostras integradas em profundidade, ao longo da secção transversal, em 3 a 7 estações espaçadas mais ou menos regu. larmente. Adicional informação sobre as amostragens e técnicas de laboratório, assin: como a relaçăo completa das medições de sedimentos em suspensão, podem encontrar-se noutra publicação (Meade et al., 1978).

(1) - U. S. Geological Survey, Denver, Colorado 80225, USA.

(2) - Hidrologia SA, Rio de Janeiro.

(3) - Companhia de Pesquisa de Recursos Minerais. Belém.

(4) -- Massachusetts Institute of Technology, Cambridge, Massachusetts 02139, USA. 


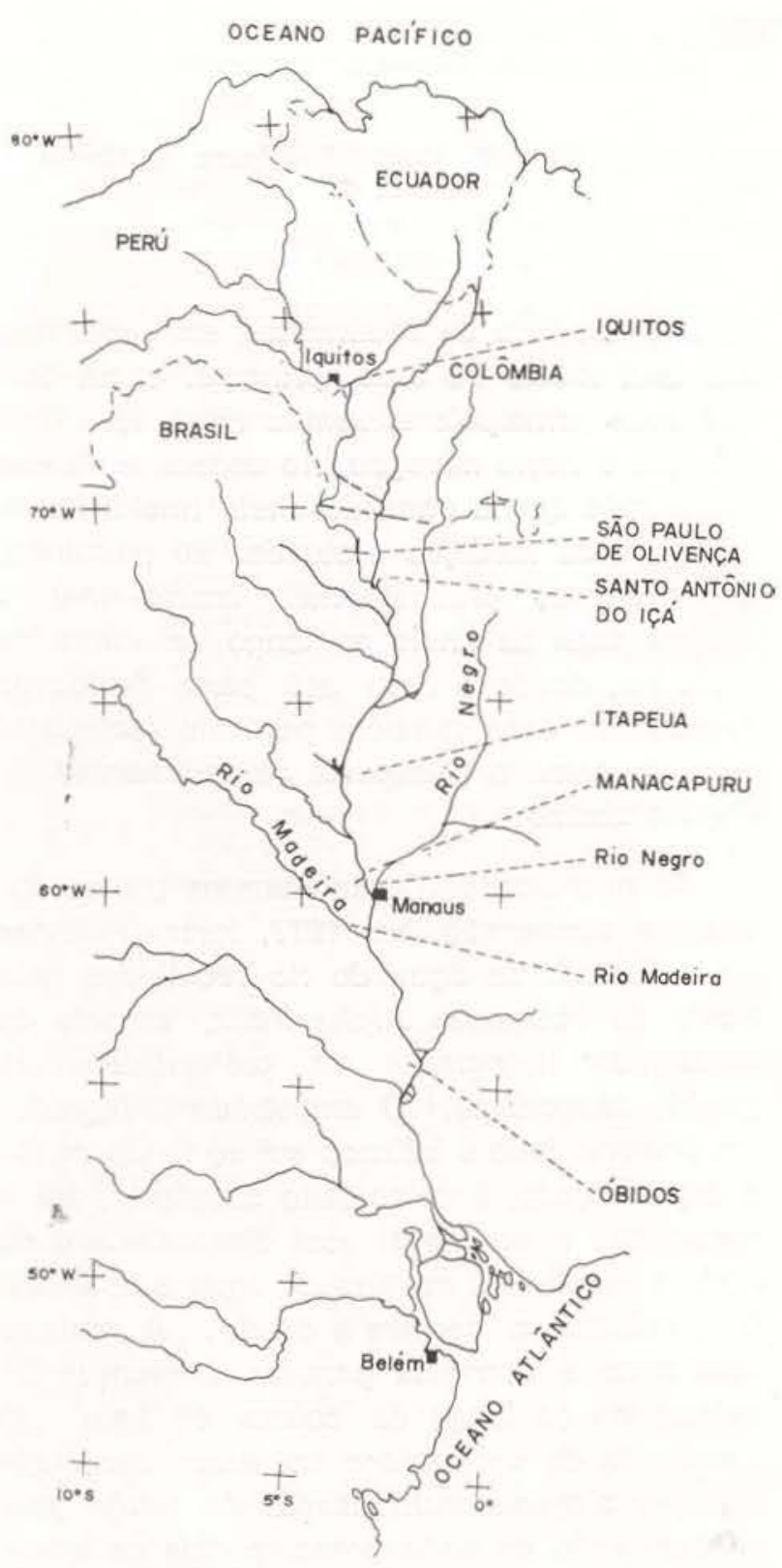

Fig. 1 - Mapa do rio Amazonas indicando as localizaçōes, no curso principal e afluentes, onde em 1977 foram medidos os transportes de sedimentos.

Os caudais indicados na Tabela 1 para as cinco secções principais do rio, no Brasil, foram estimados com base nas relaçōes níveiscaudais (curvas de vazão) que foram calculadas para estas secções pela Hidrologia S.A. e pela Companhia de Pesquisa de Recursos Minerais (CPRM). Os níveis do rio são medidos diariamente $e$ os caudais são medidos mensalmente ou bimensalmente nestas mesmas estações. Em lquitos, onde não havia medi- ções, fez-se um cálculo aproximado do caudai a partir das velocidades medidas e da área da secção transversal medida por sonar (profundidade) e radar (comprimento). O caudal do rio Madeira estimou-se a partir de medições que a Hidrologia S.A. faz mensalmente na estação de medição mais à jusante, cerca de $200 \mathrm{~km}$ a montante do local em que se recoIheram as amostras de sedimentos em suspensão. O caudal do rio Negro, sobre o qual não existem mediçōes, foi estimado por diferença entre o caudal em óbidos e em outros afluentes, medido ou estimado, conforme se indica na nota da Tabela 1.

As quantidades de sedimentos em suspensão permanecem relativamente constantes ao longo do troço entre Iquitos e Manacapu. ru, indicando que os sedimentos nesta zona do rio são provenientes dos Andes, enquanto que os afluentes que drenam a selva e entram no rio em territćrio brasileiro contribuem com grande quantidade de água mas com pequena quantidade de sedimentos adicional Este aspecto foi anteriormente notado por Sioli (1957) e foi esclarecido por Gibbs (1967), que estimou que $82 \%$ do material sólido em suspensão transportado pelo rio é proveniente de $12 \%$ da área total da bacia Amazônica situada no terreno montanhoso dos Andes. O rio Negro é o epítome dos afluentes da selva contribuindo com cerca de $20 \%$ do caudal líquido do Amazonas e apenas uma quantidade de sedimentos, relativamente trivial. Por outro lado, o rio Madeira, com as suas cabeceiras nos Andes da Bolívia, transporta uma carga razoável de sedimentos.

A diminuição, para jusante, das concentra. ções de sedimentos tinha sido anteriormente notada por Gibbs (1967) mas, os resultados que se obtiveram estão em desacordo, em diversos locais, com os dele (Fig. 2). Tanto em 1976 como em 1977, nunca se conseguiram detectar concentrações superiores a 400 $\mathrm{mg} / \mathrm{l}$ como as que Gibbs indicou durante os períodos de cheia na zona superior do rio. E, em Óbidos, na zona inferior do rio, foram medidas concentrações mais altas que as por ele executadas. A mais alta concentração de 235 mg/I, medida em Óbidos em 1977, é suportada pela informação ali recolhida no ano ante- 
TABELA 1 - Transporte de sedimentos em suspensão medidos no rio Amazonas e seus afluentes, 1977.

\begin{tabular}{|c|c|c|c|c|c|}
\hline \multirow[b]{2}{*}{ Localização } & \multirow[b]{2}{*}{ Data } & \multirow[b]{2}{*}{$\begin{array}{l}\text { Caudal } \\
\text { liquido } \\
\left(\mathrm{m}^{3} / \mathrm{s}\right)\end{array}$} & \multicolumn{3}{|c|}{ Sedimentos em suspensāo } \\
\hline & & & $\begin{array}{l}\text { Concentraçâo } \\
\text { (mg/i) }\end{array}$ & $\begin{array}{r}\text { Trar } \\
\left(\times 10^{6}\right.\end{array}$ & $\begin{array}{l}\text { ansporte } \\
\text { ton.ano ) }\end{array}$ \\
\hline Iquitos & 20 de Maio & 48000 & 400 & & 1,7 \\
\hline São Paulo de Olivença & 22 de Maio & 70000 & 275 & & 1,7 \\
\hline Santo Antonio do Içá & 23 de Maio & 80000 & 245 & & 1,7 \\
\hline Itapeúa (Coari) & 26 de Maio & 110000 & 150 & & 1,4 \\
\hline Manacapuru & 27 de Maio & 130000 & 200 & & 2,2 \\
\hline Rio Negro & 28 de Maio & $50000^{\circ}$ & 5 & & 0,02 \\
\hline Rio Madeira & 1 de Junho & 40000 & 330 & & 1,1 \\
\hline Obidos & 2 de Junho & 230000 & 235 & & 4,7 \\
\hline
\end{tabular}

a) Estimado por subtraçáo ao caudal em óbidos $230.000 \mathrm{~m} 3 / \mathrm{s}$ ) do seguinte: coudal do curso principal em Manacopuru (130.000), caudal estimado do rio Madeira e Novo Aripuaná $(40.000)$, e caudal estimado do rio Trombetas $(5.000)$ e outros afluentes $(5.000)$ entre Manacopuru e óbidos.

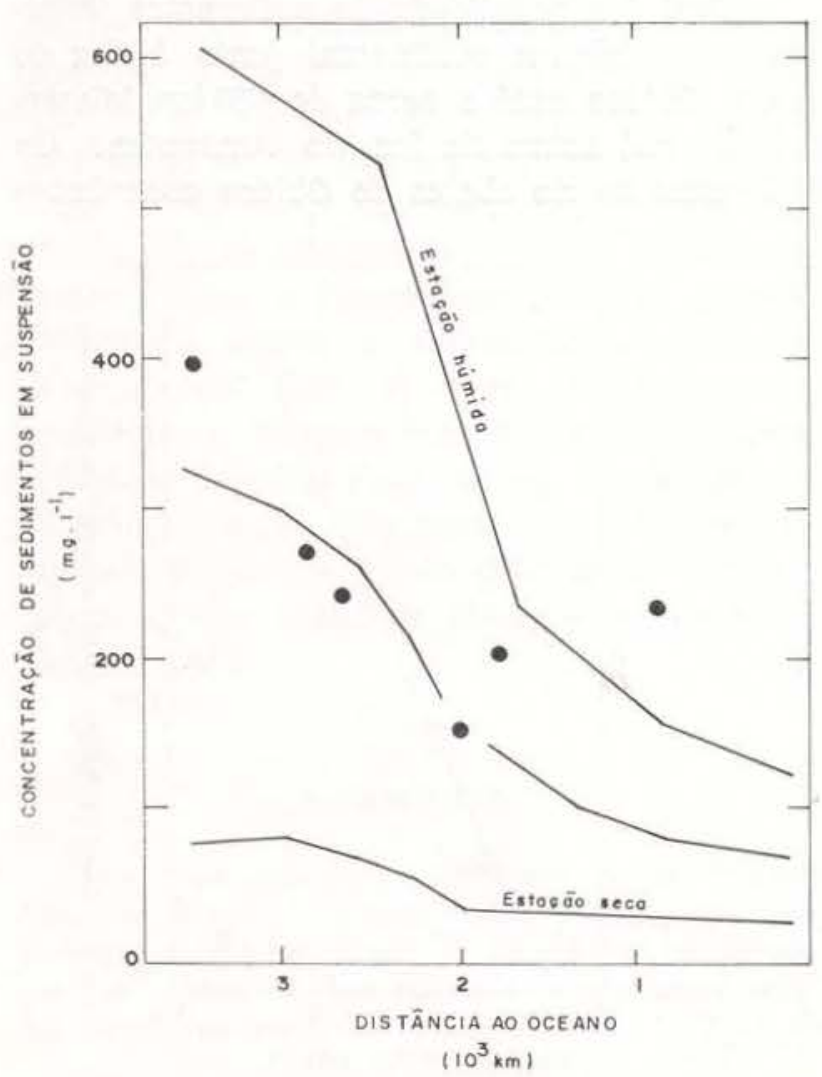

Fig. 2 - Concentraçōes de sedimentos em suspensão no curso principal do rio Amazonas. As linhas "a cheio" representam as concentraçōes indicadas por Gibbs (1967) para as estações úmidas, intermédias e secas, Os círculos negros representam as concentraçōes de material sólido em suspensāo apresentadas na Tabela 1. rior: a concentração média de material sólido em suspensão, baseada em 37 pontos ds amostragem em diferentes locais e profundidades da secção transversal, em 15 de junho de 1976 , foi entre 200 e $220 \mathrm{mg} / \mathrm{l}$. A diferença entre estes resultados e os de Gibbs está, mais provavelmente, ligada a diferenças de procedimento de amostragem.

MÉdia ANUAL DE TRANSPORTE DE SEDIMENTOS

Uma vez que os elementos da Tabela 1 representam apenas o valor diário do transporte de sedimentos, em altura de cheias, não se pode extrapolar os valores obtidos para determinar os valores globais do transporte em suspensão. Entretanto, se se combinarem os dados obtidos com outros elementos não publicados sobre a variação anual do caudal do rio com as concentrações de sedimentos em suspensão para caudais baixos, pode fazer-se uma estimativa da carga média anual de sedimentos em suspensão em Óbidos. Óbidos é a secção mais à jusante do rio onde os caudais são regularmente medidos.

Os caudais médios mensais, em Óbidos, para um período de 4 anos, são apresentados na Fig. 3A. Estas médias foram calculadas a partir de medições diárias do nivel do rio e, com menos freqüência (em geral bimensal- 
de medições do caudal do rio que foram executadas, neste período, pela CPRM e Hidrologia S.A. para o Departamento Nacional de Águas e Energia Elétrica (DNAEE).

A concentração de sedimentos em suspensão em águas baixas foi medida em óbidos em 21 de novembro de 1963 por F. C. Ames do U. S. Geological Survey (USGS) quando se concentraram os primeiros esforços para a medição do caudal do Amazonas (Oltman, 1968). O caudal do rio medido nesse dia foi de $72.500 \mathrm{~m}^{3} / \mathrm{s}$. Ames recolheu 13 amostras pontuais de sedimentos em suspensão, a diferentes profundidades e em diferentes posições ao longo da secção transversal do rio, e, calculou a concentração de material sólido em suspensão em cerca de $50 \mathrm{mg} / \mathrm{l}$.

Se, se considerar que a medição de 235 $\mathrm{mg} / \mathrm{l}$ representa a concentração de material sólido em suspensão em períodos de cheia, em Óbidos, e que as mediçōes de Ames de $50 \mathrm{mg} / \mathrm{I}$ representam a concentração em caudais baixos e, se também se considerar que a relação entre os caudais médios mensais e as concentrações médias mensais segue a lei geométrica representada na Fig. 3B, podem calcular-se os valores mensais do transporte de sedimentos, correspondentes aos caudais mádios mensais representados na Fig. $3 \mathrm{~A}$, que se podem somar, obtendo-se uma estimativa do transporte médio anual de sedimentos em suspensão em óbidos. Por este procedimento, obteve-se a estimativa de $0,93 \times 10^{9}$ ton. ano. Uma estimativa um pouco mais conservativa pode ser calculada se se admitir que a concentração média no mês de maior caudal é somente $200 \mathrm{mg} / \mathrm{l}$ e que as concentrações nos outros meses variam de forma correspondente entre este valor e a concentração mínima de $50 \mathrm{mg} / \mathrm{l}$ : neste caso, a estimativa de transporte sólido em suspensão, anual, para Óbidos é de $0,82 \times 10^{9}$ ton.ano.

Qual é a quantidade de sedimentos lançada na plataforma continental junto à foz do rio ? Óbidos está a cerca de $850 \mathrm{~km}$ (distância de rio) acima da foz do Amazonas. Os afluentes do rio abaixo de Óbidos contribuem
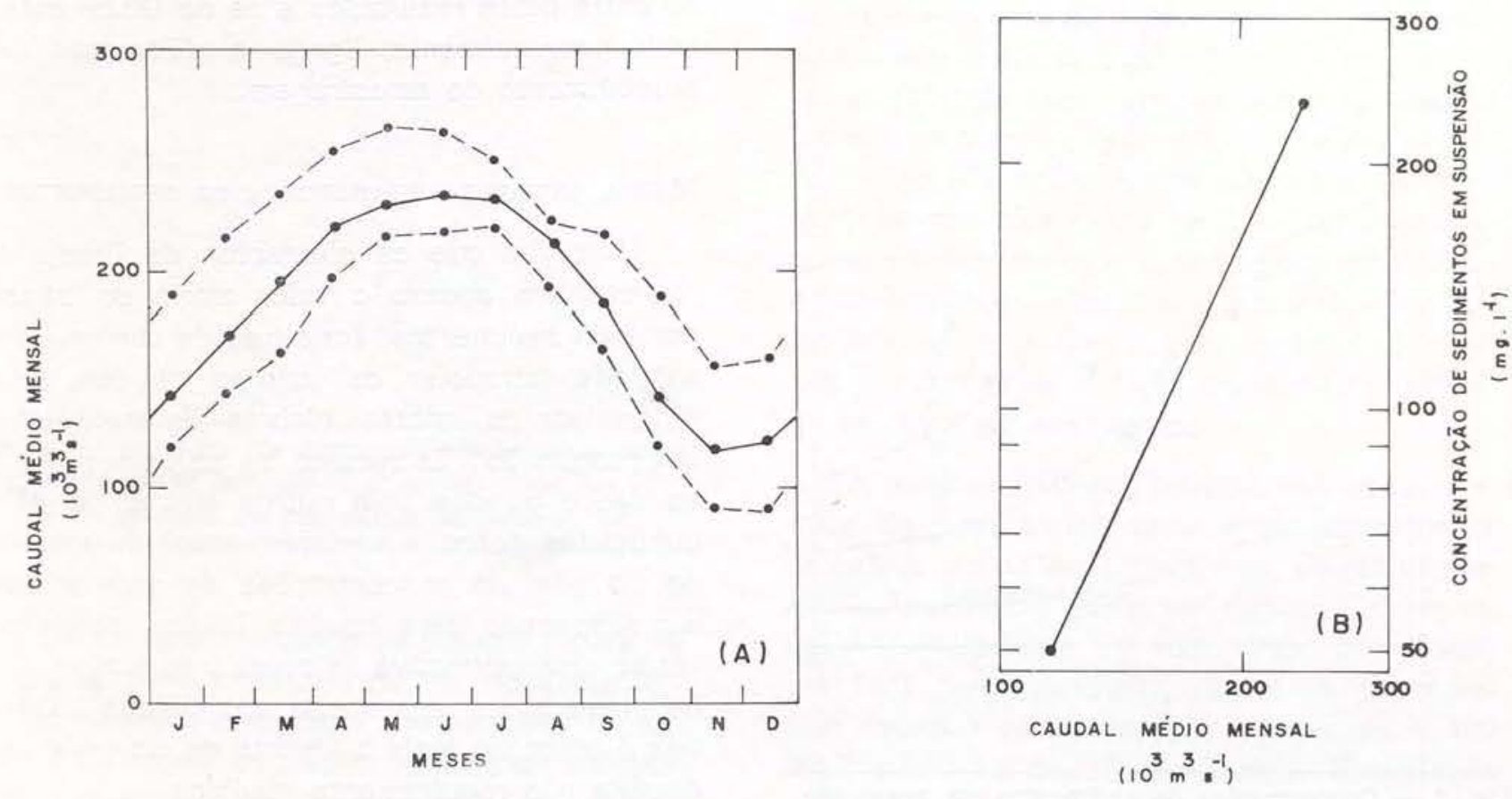

Fig. 3 - A) Caudais médios mensais do rio Amazonas em óbidos, 1971-1975. Os máximos e mínimos dos caudais médios mensais são representados pelas linhas "a tracejado"; os valores médios são indicados pela linha "a cheio". Note-se que o máximo caudal médio mensal, para o período de quatro anos, é unicamente 3 vezes maior que o mínimo. B) Relação, admitida, entre a concentração média mensal de sedimentos em suspensão e caudal médio mensal em óbidos. 
com mais $10 \%$ do caudal mas não adicionam significativa quantidade de sedimentos. A questão pōe-se em que quantidade de sedimentos é depositada na larga planície aluvionar e extenso delta que se encontra entre Óbidos e o mar. Mesmo que esta quantidade seja substancial - por exemplo, até $20 \%$ dos sedimentos que passam em Óbidos - a restante quantidade de sedimentos coloca o Amazonas logo a seguir ao rio Amarelo, na China, e ao conjunto Ganges-Bramaputra, na índia e Bangladesh, como alimentador de sedimentos para os oceanos.

\section{AGRADECIMENTOS}

Agradece-se às seguintes pessoas pela sua assistência na construção do equipamento de amostragem, obtenção de dados e revisão do manuscrito : F. C. Ames, J. P. Beverage, R. F. Hadley, D. W. Hubbell, G. A. Lutz, H. A. Mahoney, J. V. Skinner, e H. H. Stevens do USGS; oficiais e tripulação do R.V. Alpha Helix, que é operado pelo Scripps Institution of Oceanography e subsidiado pela National Science Foundation; brigadas de teodolitos da CPRM e Hidrologia S.A.; Barry Grant do MIT; Cmt. M. Perez da Marinha do Brasil; N. J. Bósio e P.M.B. Landim da Universidade Estadual Paulista, Rio Claro; Nelson da França Ribeiro dos Anjos da ELETROBRÁS; Marcelo Bezerra e Sylvio Cristino da Conceição da CPRM; Stélio da Silva Elleres de Sousa do DNAEE.

\section{SUMMARY}

From new data on the sediment in the Amazon River, we estimate the mean annual load of suspended sediment at Obidos, Brazil, to be $0.8-0.9 \times 10^{9}$ tonnes per year. Most of this sediment is discharged onto the continental shelf.

\section{BIBLIOGRAFIA}

NEDECO

1959 - River studies. Niger and Beume Amster. dam, North Holland Publishing Co., 100 p.

StrakHOV, N. M.

1961 - O nekotorykh zakonomernostiakh denudas. tii i perenosa osadochnogo materiala na ploshchadyakh gymidnykh klimatov. in Strakhov, N. M. Bezrykov, P. L., and Yablokov, V. S., editors, Soremennye osad. kt morei i oceanov: Moscow, Isdatelstovo Akad. Nauk SSSR, p. 5-27.

GisBs, R. J,

1967 - The geochemistry of the Amazon River system: Part 1. The factors that control the salinity and the composition and concentration of the suspended solids: Geological Society of America Bulletin, v. 78, no. 10, p. 1203-1232.

Holeman, J. N.

1968 - The sediment yield of major rivers of the world: Water Resources Research, v. 4, no. 4. p. $737-747$.

GUY. H. P. \& Norman, V. W.

1970 - Field methods for measurement of fluvial sediment: U. S. Geological Survey Techniques of Water-Resources Investigations, Book 3, Chap. C2, 59 p.

PORTERFIELD, G.

1972 - Computation of fluvial-sediment discharge : U. S. Geological Survey Techniques of Water-Resources Investigations, Book 3. Chap. C3, $66 \mathrm{p}$

MEAdE, R. H.; NORDIN, C. F. JR.; CURTIS, W. F.;

Mahoney, H. A. \& Delaney, B. M.

1978 - Suspended-sediment and velocity data. Amazon River and its tributaries, JuneJuly 1976 and May-June 1977; U. S. Geological Survey Open-File Report 78.

Sioli, $\mathrm{H}$.

1957 - Sedimentation im Amazonasgebiet: Geologische Rundschau. v. 45 , no. 3, p. 608-6333.

Oltman, R. E.

1968 - Reconnaissance investigations of the discharge and water quality of the Amazon River: U, S. Geological Survey Circular 552, $16 \mathrm{p}$.

(Aceito para publicaçāo em 17/05/79). 